\title{
Perioperative pregabalin and delirium following total hip arthroplasty: a post hoc analysis of a double-blind randomized placebo-controlled trial
}

\author{
Chris Farlinger, MD, MSc, FRCPC (1) - Hance Clarke, MD, PhD • Camilla L. Wong, MD, MHsC, FRCPC
}

Received: 10 July 2018/Revised: 13 July 2018/Accepted: 13 July 2018/Published online: 6 August 2018

(C) Canadian Anesthesiologists' Society 2018

\section{To the Editor,}

Postoperative delirium is associated with a risk of adverse outcomes including cognitive impairment, prolonged hospitalization, poor functional recovery, institutional placement, increased costs, and even death. ${ }^{1}$

Increased postoperative pain has been associated with the occurrence of delirium and a previous randomizedcontrolled trial (RCT) investigating parecoxib, a selective cyclooxygenase- 2 inhibitor, reduced the consumption of opioids and decreased delirium following total hip or knee replacement surgery. ${ }^{2}$ Pregabalin is a structural analogue of $\gamma$-aminobutyric acid. A meta-analysis of pregabalin for postoperative pain management found it may have a small opioid-sparing effect with a higher risk of serious adverse effects, but it is unclear if its opioid-sparing properties could also decrease postoperative delirium. ${ }^{3}$

We previously reported the results of a single centre RCT that compared perioperative pregabalin added to celecoxib with placebo with respect to pain scores and functional outcomes after elective total hip arthroplasty. ${ }^{4}$ This present report is a post hoc analysis of the postoperative delirium data from these two groups and

C. Farlinger, MD, MSc, FRCPC - C. L. Wong, MD, MHsC, FRCPC ( $\square)$

St. Michael's Hospital, Toronto, ON, Canada

e-mail: camilla.wong@utoronto.ca

H. Clarke, MD, PhD

Pain Research Unit, Toronto General Hospital, Toronto, ON, Canada

C. L. Wong, MD, MHsC, FRCPC

University Health Network, Toronto, ON, Canada

C. L. Wong, MD, MHsC, FRCPC

Li Ka Shing Knowledge Institute, Toronto, ON, Canada separate approval for this analysis was obtained from the Sunnybrook Health Sciences Centre Research Ethics Board (166-2013, approved August 2, 2013).

Inclusion and exclusion criteria, recruitment, and study duration were presented in the original publication. ${ }^{4} \mathrm{~A}$ computer-generated set of random numbers for block randomization was used, treatment allocation was concealed, and pregabalin and placebo pills were identical. All study participants, health care team members, and study personnel were blinded to the treatment assignment throughout the study. The sample size calculation was based on the estimate for the primary outcome in the original study.

Two hours prior to spinal anesthesia and surgery, all participants received $400 \mathrm{mg}$ celecoxib and were then randomized to receive $150 \mathrm{mg}$ pregabalin or placebo. Postoperatively, all participants received $200 \mathrm{mg}$ celecoxib every $12 \mathrm{hr}$ and morphine intravenously via a patientcontrolled analgesia pump for $24 \mathrm{hr}$. Beginning eight hours after the preoperative dose, participants were administered either $75 \mathrm{mg}$ pregabalin or placebo twice daily until seven days after discharge. At 08:00 a.m. on the morning after surgery, $5 \mathrm{mg}$ oxycontin was given every eight hours until the third postoperative day.

The incidence and duration of postoperative delirium were evaluated using a validated medical chart abstraction tool. ${ }^{5}$ The presence of risk factors for delirium (cognitive impairment, visual impairment, APACHE II score, and preoperative dehydration defined by a blood urea nitrogen to serum creatinine ratio of $\geq 0.1$ ) was also abstracted from the chart.

One hundred and eighty-four participants were randomized and data for 163 of the participants were analyzed (one withdrew, ten did not receive the study drug for the entire duration of study, eight stopped the study 
Table Baseline characteristics of study participants and incident delirium

\begin{tabular}{lcc}
\hline & Placebo $n=79$ & Pregabalin $n=84$ \\
\hline Demographics & & \\
Age in yr, mean (SD) & $60.0(8.9)$ & $60.1(9.3)$ \\
Female, $n(\%)$ & $39(49)$ & $41(49)$ \\
Risk factors for delirium on admission & $1(1)$ & $0(0)$ \\
Cognitive impairment, $n(\%)$ & $50(63)$ & $50(60)$ \\
Vision impairment, $n(\%)$ & $0(0)$ & $0(0)$ \\
APACHE II $>16, n(\%)$ & $12(15)$ & $9(11)$ \\
Dehydration (BUN:Cr $\geq 0.1), n(\%)$ & $74(95)$ & $83(99)$ \\
Hospitalization-related factors & $0(0)$ & $0(0)$ \\
Mode of anesthesia: spinal and sedation, $n(\%)$ & $112.2(13.3)$ & $122.7(14.5)$ \\
Intensive care unit, $n(\%)$ & $4.8(5.9)$ & $4.3(1.0)$ \\
Postoperative hemoglobin, mean (SD) & $0(0)$ & $0(0)$ \\
Length of stay in days, mean (SD) & $0(0)$ & $1(1)$ \\
Death, $n$ (\%) & & \\
Incident delirium, $n(\%) *$ & & \\
\hline APACHE Acur & &
\end{tabular}

APACHE $=$ Acute Physiologic Assessment and Chronic Health Evaluation

BUN: $\mathrm{Cr}=$ blood urea nitrogen to serum creatinine ratio

*Absolute risk $1.2 \%$; $95 \%$ confidence interval, $-2.1 \%$ to $4.5 \% ; P=1.00$

early because of an adverse event, and two had incomplete data) in an intention-to-treat analysis. The two groups $(n=$ 79 placebo and $n=84$ pregabalin) were well balanced for demographic, risk, and hospitalization variables (Table).

Only one participant developed delirium, with no difference between the placebo and pregabalin groups $(0 /$ 79 [0\%] vs 1/84 [1.2\%], respectively; absolute risk, 1.2\%; $95 \%$ confidence interval, $-2.1 \%$ to $4.5 \% ; P=1.00)$. Delirium resolved after one day.

Despite the decreases in adjunct opioid utilization and better postoperative analgesia with pregabalin that we reported previously, our study found no effect of pregabalin on postoperative delirium following elective total hip arthroplasty. All patients received a selective cyclooxygenase- 2 inhibitor perioperatively, which reduces postoperative delirium, ${ }^{2}$ and thus may have nullified any effect of pregabalin. Other limitations of this analysis include a sample size powered for the original study's primary outcome; so we were likely underpowered to examine delirium in light of its unexpectedly lower incidence. The chart abstraction method for identifying delirium is only as good as the original chart documentation. The study participants had a very low risk for postoperative delirium and these results may not apply to frailer, older individuals. Thus, it remains unclear as to the role of pregabalin as part of a multimodal pain management strategy in the risk of postoperative delirium.
Conflicts of interest None declared.

Editorial responsibility This submission was handled by Dr. Hilary P. Grocott, Editor-in-Chief, Canadian Journal of Anesthesia.

Financial support Camilla Wong is supported by the Li Ka Shing Knowledge Institute of St. Michael's Hospital. Hance Clarke is supported by a Merit Award from the Department of Anesthesia, University of Toronto. Chris Farlinger receives no financial support. Pregabalin capsules were provided by Pfizer Canada Inc.

\section{References}

1. Marcantonio ER. Postoperative delirium: a 76-year-old woman with delirium following surgery. JAMA 2012; 308: 73-81.

2. Mu DL, Zhang DZ, Wang DX, et al. Parecoxib supplementation to morphine analgesia decreases incidence of delirium in elderly patients after hip or knee replacement surgery: a randomized controlled trial. Anesth Analg 2017; 124: 1992-2000.

3. Fabritius ML, Strøm C, Koyuncu S, et al. Benefit and harm of pregabalin in acute pain treatment: a systematic review with metaanalyses and trial sequential analyses. Br J Anaesth 2017; 119: 775-91.

4. Clarke H, Pagé GM, McCartney CJ, et al. Pregabalin reduces postoperative opioid consumption and pain for 1 week after hospital discharge, but does not affect function at 6 weeks or 3 months after total hip arthroplasty. Br J Anaesth 2015; 115: 90311.

5. Inouye SK, Leo-Summers L, Zhang Y, Bogardus ST Jr, Leslie DL, Agostini JV. A chart-based method for identification of delirium: validation compared with interviewer ratings using the confusion assessment method. J Am Geriatr Soc 2005; 53: 312-8. 